\title{
Nouvelle approche pour l'estimation de l'âge des glossines par analyse d'image de l'aile
}

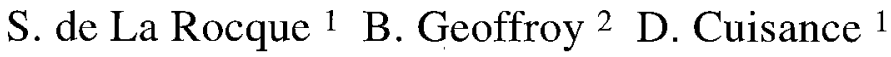

Mots-clés

Glossina tachinoides - Clossina - Age Aile - Analyse d'image.

\section{Résumé}

Une première étude sur l'évaluation de l'âge de Glossina tachinoides Westwood, 1850, par analyse des niveaux de gris de l'aile est réalisée. Sur des lots de femelles provenant du laboratoire, cinq groupes apparaissent significativement différents. A condition d'être validée sur des glossines naturelles, cette méthode pourrait constituer une nouvelle approche de ce facteur important dans l'épidémiologie des trypanosomoses animales.

\section{INTRODUCTION}

La connaissance de l'âge des glossines est un élément important pour étudier l'épidémiologie des trypanosomoses, évaluer l'efficacité des campagnes de lutte antivectorielle et analyser les dynamiques de population de ces insectes. Différentes techniques de détermination ont été décrites, certaines fondées sur l'examen de structures morphologiques exlernes : usure des ailes (4), couleur de l'abdomen, capacité de dévagination du ptilinum par pression, élasticité du tégument thoracique, d'autres sur l'examen de structures morphologiques internes: stade de maturation des ovaires (2, 3,8 ), présence du sac intestinal résiduel et longueur de la membrane péritrophique pour les glossines ténérales (6), ou d'autres encore sur le dosage de substances physiologiques (taux de ptéridine) $(5,7)$. Cependant ces méthodes sont peu précises ou longues et fastidieuses pour une utilisation sur le terrain.

Dès 1950, Squire avait imaginé d'utiliser la couleur des ailes comme critère d'évaluation de l'âge (9). Il regroupait les glossines en trois groupes selon la teinte de leurs ailes, allant du fumé pour les jeunes mouches au fauve pour les plus âgées (1). Les techniques actuelles d'analyse d'images ont incité les auteurs à approfondir cette idée, pour tenter de mettre au point un outil rapide et simple de détermination de l'âge des glossines.

\section{MATERIEL ET METHODES}

Des glossines femelles (Glossina tachinoides Westwood, 1850) d'âge connu, provenant de l'élevage du CIRAD/ORSTOM de Montpellier ont été utilisées. Leurs ailes sont montées à sec entre lame et lamelle, puis examinées sous une loupe binoculaire (grossissement 50). Une caméra, reliée à un ordinateur (logiciel

1. CIRAD-EMVT, Campus international de Baillarguet, BP 5035, 34032 Montpellier Cedex 1, France

2. ORSTOM, Département Santé, BP 5045, 34032 Montpellier Cedex 1, France
Optimas $\left({ }^{\circledR}\right)$ permet de fixer l'image sur l'écran, puis d'analyser le niveau de gris obtenu par transparence.

La surface étudiée doit être suffisamment grande pour éviter une trop importante variabilité induite par les différentes microstructures alaires (notamment les microtriches). Dans cette étude, un disque de $0,5 \mathrm{~mm}$ de diamètre situé sous la base de la cellule en "hachette", devant l'intersection de la nervure longitudinale $V$ et de la nervure transverse postérieure (figure 1) a été retenu comme surface d'analyse.

Huit sćries d'ailes ont ćté cxaminées, correspondant à des glossines âgées de $0,5,10,14,19,26,40$ et 55 jours et comprenant respectivement $31,24,23,24,22,24,24$ et 27 échantillons. Pour chaque aile, a été relevé d'une part la valeur moyenne du niveau de gris, et d'autre part l'amplitude des variations de gris dans l'aire de mesure (différence entre les valeurs des pixels les plus sombres et les plus clairs). Les moyennes sont comparées par analyse de variance, puis deux à deux (test de $\mathrm{t}$ ). Les variances sont comparées par le test de F. Le risque choisi est de 5 p. 100 pour tous les tests.

Les valeurs de gris obtenues sont étroitement dépendantes des caractéristiques de la lumière incidente. Pour pallier à cet inconvénient, les valeurs retenues correspondent en fait à la différence entre un niveau "zéro" obtenu en l'absence d'aile et la valeur affichée.

\section{RESULTATS}

Lorsque la glossine est au repos, l'aile gauche recouvre la droite dans les deux tiers des cas, quel que soit l'âge de l'insecte ( $S$. de La Rocque, non publié). Cette observation est à prendre en considération car l'aile supérieure est exposée aux souillures exogènes, souvent importantes dans des conditions d'élevage. Les analyses ont donc été réalisées sur les ailes inférieures.

Jusqu'à 12 paires d'ailes peuvent être montées sur une même lame. Il a été vérifié que la technique et le matériel de montage 


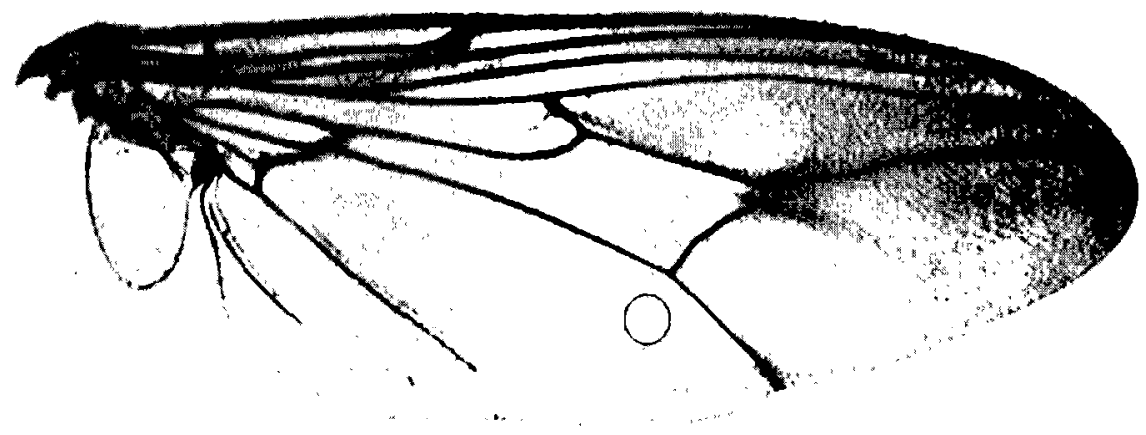

Figure 1: position de l'aire circulaire de mesure (petit cercle) sur l'aile de Glossina tachinoides.

n'induisait pas de variation entre les lames au sein de la même classe d'âge.

\section{Variation du niveau de gris moyen avec l'âge}

Lors de cette étude, cinq classes d'âge significativement différentes ont pu être séparées : $\mathrm{J}_{0}, \mathrm{~J}_{10^{-}} \mathrm{J}_{14}, \mathrm{~J}_{19}, \mathrm{~J}_{26^{-}} \mathrm{J}_{40}$ et $\mathrm{J}_{55}$. La classe $\mathrm{J}_{5}$ est intermédiaire entre les classes $\mathrm{J}_{0}$ et $\mathrm{J}_{10^{-}} \mathrm{J}_{14}$, dont elle ne diffère pas au risque choisi.

Le niveau de gris moyen évolue avec l'âge selon trois phases distinctes (figure 2) : il diminue durant les deux semaines qui suivent l'éclosion (différences significatives entre $\mathrm{J}_{0}$ et $\mathrm{J}_{10^{-}} \mathrm{J}_{14}$ ), puis augmente fortement et régulièrement entre la deuxième et la quatrième semaine (différences significatives entre $\mathbf{J}_{10^{-}} \mathrm{J}_{14}, \mathbf{J}_{19}$ et $\mathrm{J}_{26} \mathrm{~J}_{40}$ ), pour se stabiliser en plateau légèrement ascendant par la suite (différences significatives entre $\mathrm{J}_{26}-\mathrm{J}_{40}$ et $\mathrm{J}_{55}$ ).

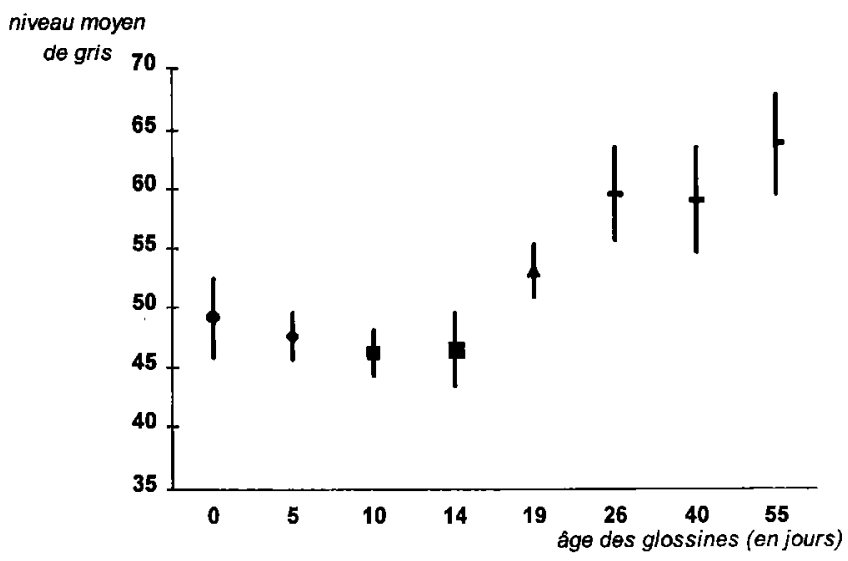

Figure 2 : évolution du niveau de gris moven avec l'âge (les valeurs indiquées correspondent à la différence entre un "niveau 0 " valant 255 et la valeur obtenue).

\section{Amplitude des variations de gris à l'intérieur des disques}

Les moyennes de l'amplitude des variations de gris dans l'aire circulaire choisie ne diffèrent pas significativement selon les classes d'âge : le contraste entre les microstructures alaires les plus sombres et les plus claires reste donc constant. La classe $\mathrm{J}_{0}$ fait exception, avec une amplitude plus faible que celle de toutes les autres classes d'âge.

\section{DISCUSSION}

Juste après l'éclosion de l'adulte, les ailes sont gris-foncé, et teintées de manière homogène. Dans les jours qui suivent, le niveau de gris moyen diminue, les ailes s'éclaircissent globalement. En même temps les microtriches s'assombrissent, ce qui augmente le contraste entre les microstructures de l'aile et donc l'amplitude des variations de gris. Ensuite ce contraste reste constant, mais l'aile devicnt globalement plus grise, rapidement dans un premier temps, plus lentement ensuite.

Les écarts-types autours des moyennes de niveau de gris sont élevés. Ils pourraient certainement être réduits par une meilleure maîtrise dans la constance de la lumière incidente. Les résultats seront certainement plus probants lorsque le niveau d'autres couleurs pourra être analysé. Le jaune semble, à vue d'oeil, évoluer nettement avec l'âge.

Cette approche est cependant prometteuse pour le développement d'une méthode simple et rapide qui permettrait de distinguer cinq classes d'âge chez les glossines à partir de la couleur des ailes. Ces classes sont particulièrement intéressantes car il est admis que ce sont essentiellement les mouches ténérales qui s'infectent, et que le cycle des parasites dans l'insecte dure justement le temps de passer d'une phase à l'autre (environ 10 jours pour Trypanosoma vivax, 14 jours pour T. congolense et 30 jours pour T. brucei).

Cette première étude a été réalisée avec des glossines d'élevage sur une seule espèce ( $G$. tachinoides). Elle demande à être confirmée sur des mouches in natura dont l'âge est connu, soit par des lâchers de lots marqués et recapturés, soit par référence à l'âge physiologique chez des femelles disséquées. La méthode mérite également d'être appliquée à d'autres espèces ou sous espèces de glussines.

\section{BIBLIOGRAPHIE}

1. BUXTON P.A., 1955. The natural history of tsetse flies. London, United Kingdom. London School of Hygiene and tropical Medicine, H.K. Lewis, 816 p. (Collection Memoirs No. 10)

?. CHALIIFR A., 1965. Amélioration de la méthode de détermination de l'âge physiologique des glossines. Etudes faites sur Clossina palpalis gambiensis Vanderplank, 1949. Bull. Soc. Path. exot., 58 : 250-259.

3. ITARD J., 1966. Cycle de l'oogénèse chez les femelles de Glossina tachinoides West. et détermination de l'âge physiologique. Revue Elev. Néd. vét. Pays trop., $19: 331-350$.

4. JACKSON C.H.N., 1946. An artificially isolated generation of tsetse flies. Bull. ent. Res., 37: 291-299. 
5. LANGLEY P.A., HALL M.J.R., FELTON T., 1988. Determining the age of tsetsc flics, Glossina spp. (Diptcra: Clossinidae): an appraisal of the pteridine fluorescence technique. Bull. ent. Res., 78: 387-395.

6. LAVEISSIERE C., 1975, Détermination de l'âge des glossines ténérales (Glossina tachinoides Westwood). Cah. ORSTOM, Sér. Ent. méd. Parasitol., 13 : 3-11.

\section{Summary}

De La Rocque S., Geoffroy B., Cuisance D. A new approach to estimate tsetse fly ages by image analyzing of the wings

A first study has been performed to determine the age of Glossina tachinoides Westwood, 1850 by analyzing the various levels of grey in the wing. Among the laboratory female tsetse flies studied, five groups stood out as significantly different. This method could constitute a new approach of this important factor in the study of animal trypanosomosis epidemiology, provided it is confirmed with non-laboratory tsetse flies.

Key words: Glossina tachinoides - Clossina - Age - Wing Image analysis.
7. LEHANE M.J., MAIL T.S., 1985. Determining the age of adult male and female Glossina morsitans morsitans using a new technique. Ecol. Entomol., 10: 219-224.

8. SA.UNDERS D.S., 1960. Determination of physiological age for female Glossina morsitans. Nature, 186: 651.

9. SQUIRE F.A., 1950. Age-grouping of tsetse flies as an aid in the study of their bionomics. Nature, 165: 307-308.

Reçu le 8.1.96, accepté le 18.6.96

\section{Resumen}

De La Rocque S., Geoffroy B., Cuisance D. Nuevo enfoque para la estimación de la edad de las glosinas mediante el análisis de la imagen del ala

Se realizó un primer estudio sobre la evaluación de la edad de la Clossina tachinoides Westwood, 1850 mediante el análisis de lós niveles de gris en el ala. Entre grupos de hembras provenientes de laboratorio, se distinguen cinco grupos significativamente diferentes. Con la condición de una previa verificación sobre glosinas naturales, este método podria representar un nuevo enfoque para el esludio de este importante factor en la epidemiología de los tripanosomas animales.

Palabras clave : Glossina tachinóides - Glossina - Edad - Ala Análisis de la imagen. 